\title{
Brain regions underlying repetition and auditory-verbal short-term memory deficits in aphasia: Evidence from voxel-based Lesion symptom mapping
}

\author{
Juliana V. Baldo ${ }^{1}$, Shira Katseff ${ }^{1,2}$, and Nina F. Dronkers ${ }^{1,3,4}$ \\ ${ }^{1}$ VA Northern California Health Care System, Martinez, CA, USA \\ ${ }^{2}$ University of California, Berkeley, CA, USA \\ ${ }^{3}$ University of California, Davis, CA, USA \\ ${ }^{4}$ University of California, San Diego, CA, USA
}

Background: A deficit in the ability to repeat auditory-verbal information is common among individuals with aphasia. The neural basis of this deficit has traditionally been attributed to the disconnection of left posterior and anterior language regions via damage to a white matter pathway, the arcuate fasciculus. However, a number of lesion and imaging studies have called this notion into question.

Aims: The goal of this study was to identify the neural correlates of repetition and a related process, auditory-verbal short-term memory (AVSTM). Both repetition and AVSTM involve common elements such as auditory and phonological analysis and translation to speech output processes. Based on previous studies, we predicted that both repetition and AVSTM would be most dependent on posterior language regions in left temporo-parietal cortex.

Methods \& Procedures: We tested 84 individuals with left hemisphere lesions due to stroke on an experimental battery of repetition and AVSTM tasks. Participants were tested on word, pseudoword, and number-word repetition, as well as digit and word span tasks. Brain correlates of these processes were identified using a statistical, lesion analysis approach known as voxel-based lesion symptom mapping (VLSM). VLSM allows for a voxel-by-voxel analysis of brain areas most critical to performance on a given task, including both grey and white matter regions.

Outcomes \& Results: The VLSM analyses showed that left posterior temporo-parietal cortex, not the arcuate fasciculus, was most critical for repetition as well as for AVSTM. The location of maximal foci, defined as the voxels with the highest $t$ values, varied somewhat among measures: Word and pseudoword repetition had maximal foci in the left posterior superior temporal gyrus, on the border with inferior parietal cortex, while word and digit span, as well as number-word repetition, were centred on the border between the middle temporal and superior temporal gyri and the underlying white matter.

Conclusions: Findings from the current study show that (1) repetition is most critically mediated by cortical regions in left posterior temporo-parietal cortex; (2) repetition and AVSTM are mediated by partially overlapping networks; and (3) repetition and AVSTM

Address correspondence to: Juliana V. Baldo, 150 Muir Rd. (126R), Martinez, CA 94553, USA. E-mail: juliana@ebire.org

This research was supported in part by the Department of Veterans Affairs Research \& Development, NIH/NINDS 5 P01 NS040813, and NIH/NIDCD 5 R01 DC00216. We would like to thank Brenda Redfern and Johnna Shapiro for their assistance in the design of the stimuli. We are also very thankful to the research volunteers who took part in this study.

\footnotetext{
(C) 2011 Psychology Press, an imprint of the Taylor \& Francis Group, an Informa business http://www.psypress.com/aphasiology

DOI: $10.1080 / 02687038.2011 .602391$
} 
deficits can be observed in different types of aphasia, depending on the site and extent of the brain injury. These data have implications for the prognosis of chronic repetition and AVSTM deficits in individuals with aphasia when lesions involve critical regions in left temporo-parietal cortex.

Keywords: Repetition; Short-term memory; Aphasia; Conduction aphasia; Temporal cortex; Parietal cortex.

The ability to repeat verbal information involves a number of cognitive processes, from auditory processing and phonological analysis to output mapping and speech production. Deficits in repetition are commonly associated with the syndrome of conduction aphasia, which is characterised by relatively good comprehension and fluent but paraphasic speech, accompanied by relatively poor repetition abilities (Goodglass, 1992; Kohler, Bartels, Herrmann, Dittmann, \& Wallesch, 1998). However, repetition deficits are common to different types of aphasia and necessary for diagnosis of not only conduction aphasia but also Wernicke's and Broca's, for example (Brown, 1975). In this paper we investigate the anatomical locus of this common behavioural deficit, along with a related cognitive process, auditory-verbal short-term memory, using a voxel-based lesion symptom mapping approach.

Based on Wernicke's (1906) model of language as updated by Geschwind (1972), it is commonly held that the ability to repeat auditory-verbal information is mediated by the arcuate fasciculus (for reviews, see Bernal \& Ardila, 2009; Catani \& Mesulam, 2008). The arcuate fasciculus is a fibre bundle that, along with the superior longitudinal fasciculus, runs longitudinally within the cerebral hemisphere and connects portions of the middle and superior temporal gyri with anterior cortical regions (Catani, Jones, \& Ffytche, 2005; Catani \& Mesulam, 2008; Petrides \& Pandya, 1988; Turken \& Dronkers, 2011). This conceptualisation of repetition predicts that individuals with impaired repetition, such as those with conduction aphasia, should have underlying damage to the arcuate fasciculus. A close look at the research literature, however, shows that this association between the arcuate fasciculus and repetition has not been consistently upheld (Axer, Keyserlingk, Berks, \& Keyserlingk, 2001; Bartha \& Benke, 2003; Bernal \& Ardila, 2009; Brown, 1975; Kempler et al., 1988; Mendez \& Benson, 1985; Selnes, van Zijl, Baker, Hillis, \& Mori, 2002; Shuren et al., 1995). For example, intracranial stimulation studies have shown that stimulation of posterior cortex - specifically, the superior temporal gyrus (STG) and inferior parietal cortex (IP) - is sufficient to induce symptoms such as impaired repetition with intact comprehension (Anderson et al., 1999; Quigg \& Fountain, 1999; Quigg, Geldmacher \& Elias, 2006), and that stimulation of the arcuate fasciculus alone results in anomia, not impaired repetition (Duffau et al., 2002). Furthermore, resection of portions of the arcuate fasciculus/superior longitudinal fasciculus is possible without significant reduction in word repetition ability (Shuren et al., 1995), and Selnes et al. (2002) described an individual with a lesion in the left arcuate fasciculus who had normal sentence repetition (but see Breier, Hasan, Zhang, Men, \& Papanicolaou, 2008). Even the oft-cited early lesion studies by Geschwind and colleagues (e.g., Benson et al., 1973) and Damasio and Damasio (1980) found that lesions in cortical regions such as STG/IP cortex could result in the presentation of conduction aphasia and concomitant repetition deficits. Similarly, a review by Green and Howes (1977) found that, in 25 cases of conduction aphasia, the most common lesion site was the left supramarginal gyrus (part of IP cortex), followed by the left STG. Last, a more recent 
study by Bartha and Benke (2003) of 20 cases of conduction aphasia with significant repetition deficits found that lesions were clustered either in left temporo-parietal cortex or in posterior inferior temporal cortex.

Functional neuroimaging studies have also suggested that repetition/conduction aphasia is associated with posterior peri-Sylvian cortex. Fridriksson et al. (2010) assessed the neural correlates of simple repetition in a group of 39 individuals with acute left hemisphere strokes, using both perfusion imaging and structural MRI data. The more functional technique, perfusion imaging, showed that impaired repetition was associated with cortical hypoperfusion in the left inferior supramarginal gyrus and temporo-parietal junction. The structural data, however, showed an association between impaired repetition and lesions in the white matter medial to the left supramarginal gyrus. Kempler et al. (1988) used positron emission tomography (PET) to study 10 individuals with conduction aphasia who had lesions primarily in left temporo-parietal cortex. They reasoned that if conduction aphasia were truly a disconnection syndrome, then posterior, structural damage should result in hypometabolism in frontal targets (e.g., Broca's area). They found that only $50 \%$ of individuals showed this pattern. The other individuals showed no evidence of frontal hypometabolism. They suggested that conduction aphasia is not a disconnection syndrome but rather results from damage to perisylvian regions and that, depending on how anterior the damage extends, individuals may appear more "Broca-like" or "Wernicke-like" (p. 279). Repetition deficits, then, are not conclusively linked to arcuate fasciculus damage.

A better understanding of the neural correlates of repetition in aphasia may emerge from a comparison with more general impairments in auditory-verbal short-term memory (AVSTM; Caramazza, Basili, Koller, \& Berndt, 1981; Shallice \& Warrington, 1977). AVSTM refers to a temporary store of verbal information, which is available for retrieval over a very short period, on the order of seconds. Like repetition, AVSTM requires phonological input processing and translation to speech output processes, but with an additional storage/rehearsal load. AVSTM is often affected in individuals with aphasia and is tested by immediate recall of information, for example, in span tasks that measure the number of items such as digits or words that can be repeated (Martin \& Ayala, 2004). The idea that AVSTM could be selectively affected and dissociated from long-term memory was confirmed by Warrington and colleagues who described a series of cases in which neurologic patients had significantly reduced memory spans and impaired repetition (Shallice \& Warrington, 1977; Warrington, Logue, \& Pratt, 1971; Warrington \& Shallice, 1969). In these cases performance did not improve when individuals were allowed to respond non-verbally (e.g., pointing), showing that the deficit was not simply an output problem but a central disruption of AVSTM.

Case studies of such AVSTM deficits came to be framed in terms of Baddeley's working memory model, which includes mechanisms for both auditory-verbal and visuospatial working memory (Baddeley, 2003; Baddeley \& Hitch, 1994; Belleville, Peretz, \& Arguin, 1992; Shallice \& Butterworth, 1977; Vallar \& Baddeley, 1984; Vallar, Di Betta, \& Silveri, 1997; Warrington et al., 1971). Most germane to the current paper, Baddeley's model proposes that auditory-verbal working memory has two components: a phonological store, responsible for very brief storage of verbal information (on the order of seconds), and an articulatory rehearsal component, responsible for refreshing that information and keeping it active (Baddeley, 2000, 2003; Baddeley \& Hitch, 1994). 
While early cases provided evidence of cognitive dissociations with respect to AVSTM, more recent studies using modern neuroimaging techniques have been able to systematically test the notion that short-term memory and its component parts are associated with distinct brain regions or networks (Baldo \& Shimamura, 2000; Cohen et al., 1997; D’Esposito et al., 1995; Gruber, 2001; Jonides et al., 1998; Owen, McMillan, Laird, \& Bullmore, 2005; Petrides, Alivisatos, Meyer, \& Evans, 1993; Ravizza, Delgado, Chein, Becker, \& Fiez, 2004). In a meta-analysis of lesion studies, D'Esposito and Postle (1999) found that impairments on delayed response tasks, indicative of disruption in the rehearsal component of AVSTM, were associated with lesions in pre-frontal cortex (PFC). A small subset of the studies in the meta-analysis included individuals with left posterior lesions (e.g., left temporo-parietal lesions). Unlike the individuals with PFC lesions, individuals with left posterior lesions exhibited deficits on span tasks, indicating a selective disruption of the phonological store. More recently, Baldo and Dronkers (2006) directly compared performance on a series of span and rehearsal tasks in a small group of individuals with focal lesions due to stroke either in inferior parietal (IP) or inferior frontal (IF) cortex. Tasks requiring the phonological store (span tasks, auditory rhyming, and repetition) were disproportionately impaired in the individuals with IP lesions. Individuals with IF lesions, on the other hand, performed like controls on these phonological store tasks, but were disrupted on tasks that involved articulatory rehearsal (e.g., visual rhyming). Functional neuroimaging studies have also reported similar networks subserving these components of AVSTM (Hickok, Buchsbaum, Humphries, \& Muftuler, 2003; Honey, Bullmore, \& Sharma, 2000; Jonides et al., 1998; Postle, Berger, \& D’Esposito, 1999).

In short, previous studies of AVSTM have often implicated left temporal and parietal regions, especially on tasks specifically tapping the phonological store component. The neural basis of repetition, on the other hand, has traditionally been attributed to white matter pathways connecting posterior and inferior language regions. In the current study we used voxel-based lesion symptom mapping (VLSM), which allowed us to directly compare the neural correlates of repetition and AVSTM in a large group of individuals with left hemisphere lesions. VLSM is a statistical technique that allows for a voxel-by-voxel analysis of the whole brain. VLSM differs from functional imaging studies, in that VLSM identifies brain regions that are most critically associated with task performance, while functional imaging tasks highlight the range of brain regions that are recruited/involved in a task. Given the literature described above, one of the central questions was whether repetition deficits would be primarily associated with lesions in STG/IP or rather in the underlying white matter, namely the arcuate fasciculus. A second question we addressed was whether repetition and AVSTM would be associated with overlapping anatomic correlates, given that these abilities share a number of processing elements. Individuals were tested on an experimental battery that included several different subtests: repetition of single words, non-words, and number-words, as well as span tasks to test AVSTM, specifically the phonological store component. For each condition, examinees were tested on items of gradually increasing length (e.g., increasing number of syllables in word repetition and increasing list length in word/digit span). This study was novel in that it involved a very large sample of individuals tested on a series of repetition and STM tasks, and these data were then combined with structural lesion data and subjected to a statistical, voxel-based technique for analysis of anatomic correlates. Based on the preponderance of research, we predicted that overall performance on the repetition/span 
battery would be most dependent on the left STG/IP cortex and that repetition and AVSTM would both be dependent on overlapping areas in this region.

\section{METHOD}

\section{Participants}

The participants were 84 (23 female) individuals who had suffered a single, left hemisphere cerebrovascular accident (i.e., stroke). Inclusion criteria included being native English-speaking, right-handed, with current brain imaging available and no prior neurologic, psychiatric, or substance abuse history. Individuals were all tested in the chronic phase of their stroke (at least 12 months post-injury), so that symptoms were relatively stable ( $M=56.8$ months post-injury; $S D=54.8)$. The average age of the sample was 60.3 years $(S D=11.2)$, and the average education was 14.7 years $(S D=3.2)$.

Based on the Western Aphasia Battery (WAB; Kertesz, 1982), the sample included individuals with Wernicke's aphasia $(n=6)$, Broca's aphasia $(n=13)$, conduction aphasia $(n=7)$, anomic aphasia $(n=18)$, unclassifiable aphasia $(n=7)$, and individuals who fell within normal limits (WNL; $n=33$ ). The WNL designation is given to individuals who score at least 93.8 out of 100 on the WAB; however, most of these individuals still have clinically significant word-finding deficits that are too mild to be detected by the WAB. The average score on the WAB was 79.7 out of $100(S D=22.3$; range: 18.9-100). This wide range of aphasia severity was critical for the current study, as it provided a large sample of individuals with and without repetition deficits that could be compared both behaviourally and anatomically with VLSM analyses. Several additional individuals with severe aphasia (e.g., global aphasia) were not able to comply with task instructions on the experimental measures and thus were not included in the study. The determination to discontinue testing in such individuals was made by examiners who were unaware of the goals/predictions of the current study. Individuals with primarily motor speech disorders (e.g., apraxia of speech) were not part of the participant pool for this study.

\section{Materials and Procedures}

Behavioural measures. Participants were tested on a series of language and neuropsychological measures as part of a larger research protocol. The main language measure was the WAB and included subtests that measured distinct speech and language functions such as fluency, comprehension, repetition, and naming. The main experimental measure was a repetition/span battery with several subtests designed in-house to parallel the digit span task of the Wechsler Adult Intelligence Scale. Examinees were asked to repeat an auditorily presented item, and if the item was repeated correctly the next item of increased length was presented. If repetition of the item failed another item of the same length was presented, and if this item was failed the subtest was discontinued. The battery included the following subtests: (1) repetition of single words, number-words, and pseudowords (e.g., kabit) of increasing number of syllables; (2) a word span task (lists of increasing numbers of words); and (3) a digit span task (i.e., lists of increasing number of digits). Scoring was based on the maximum length the examinee could repeat/recall on at least one of the two trials (i.e., the maximum number of syllables for word, number, and pseudoword repetition and 
the maximum list length for the span tasks). Minor distortions due to motor speech symptoms such as dysarthria were counted as correct if the examiner could determine the item was accurately recalled/repeated.

Lesion analysis. Participants' lesions were imaged with 3D MRI scans, or with 3D CT, if MRI was contra-indicated. Although CT is not as sensitive as MRI, it provides adequate information to delineate the major extent of the lesion and allowed us to include a larger sample. Lesions were either traced directly on the digital brain images using MRIcro (Rorden \& Brett, 2000) or were drawn onto standardised brain templates by a board-certified neurologist who was blind to the participants' behavioural presentation. In the former case, lesions were drawn on the individual's T1 MRI image in native space and then registered with the MNI template using the standard nonlinear spatial normalisation procedure from SPM2 (Statistical Parametric Mapping, Wellcome Trust Centre for Neuroimaging). A cost function masking procedure was used to avoid distortions due to the lesion itself (Brett, Leff, Rorden, \& Ashburner, 2001). In the latter case the template brain was manually transformed to a commonly used single individual's brain in MNI space. This transformation was non-linear and was determined slice by slice by matching manually selected control points in the two brains using a local weighted mean transformation implemented by the cpselect, cp2tform, and imtransform functions in MATLAB 6.5 (Mathworks, Natick, MA). Figure 1 shows an overlay of the participants' lesions. As can be seen, the maximal lesion overlap (in $51 \%$ of individuals) was centred in left fronto-insular cortex. The average lesion volume was $97.6 \mathrm{cc}(S D=79.5)$.

To determine the anatomic correlates of the repetition/span tasks used in the current study we used voxel-based lesion symptom mapping (VLSM; for a review, see Baldo, Wilson, \& Dronkers, in press; Bates et al., 2003). In VLSM a $t$-test is used to compare performance on every measure in individuals with a lesion versus individuals without a lesion in each voxel. In other words, for any particular voxel, a $t$-test is run with lesion status as the independent variable (lesioned or not) and behavioural performance as the dependent variable. For the present analysis, $t$-tests were confined to those voxels in which there were at least five individuals with a lesion and five individuals without a lesion. This adjustment was made to avoid spurious $t$-test

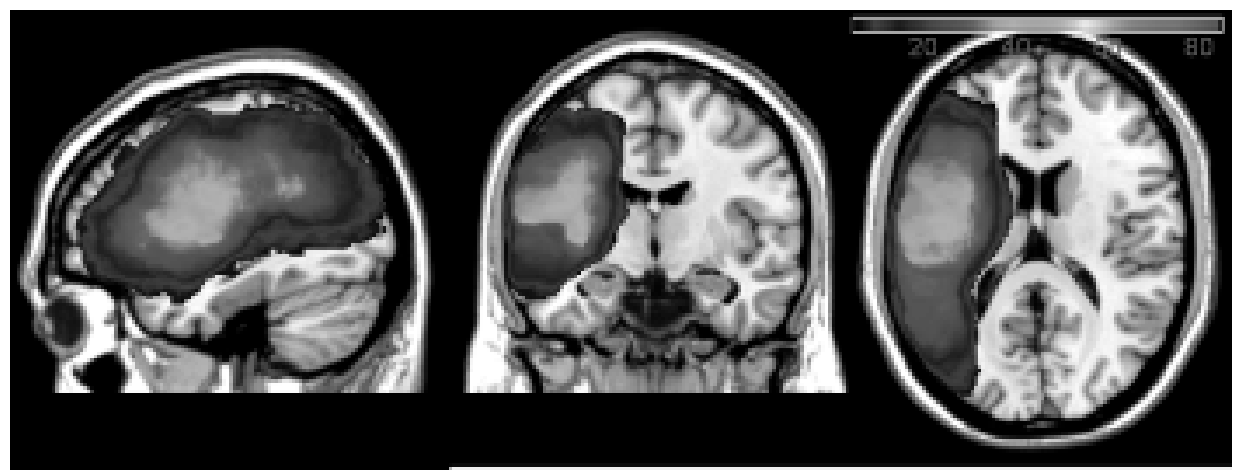

Figure 1. Overlay of participants' reconstructed lesions. This figure depicts only those voxels with a minimum of at least five individuals' lesions (shown in purple), which was the minimum number of individuals per voxel necessary to be included in the VLSM analyses to protect against spurious findings. 
results for voxels in which very few individuals had lesions. A statistical threshold cut-off ( $t$-value) with alpha set at .05 was determined based on permutation testing $(n=1,000)$, a conservative multi-comparison correction method (see Kimberg, Coslett, \& Schwartz, 2007). In this method each permutation test randomly re-pairs the set of lesioned voxels with the set of behavioural scores and re-runs the $t$-tests across all voxels; 1000 of these tests are used to determine how often high $t$ values appear by chance. Only the top 5\% of $t$-values are considered significant, and only voxels exceeding these cut-offs are shown in the VLSM maps in the Results below.

Prior to running the VLSM analyses, we generated a map to determine the distribution of statistical power for the current sample, based on a large effect size (0.8) and an alpha of .05 (Cohen, 1988, 1992; Kimberg et al., 2007). There was sufficient power in the left peri-Sylvian regions and throughout much of the left hemisphere. This included the areas of greatest interest for this study, the left STG / IP cortex and arcuate fasciculus. Power in these regions exceeded a minimum threshold of 0.8 .

\section{RESULTS}

\section{Behavioural performance}

Participants' overall performance (percent correct) on the experimental repetition/span battery was as follows: Individuals who were within normal limits (WNL) performed best (90.4\% correct), followed by individuals with anomic aphasia $(80.0 \%$ correct), unclassifiable aphasia $(75.0 \%$ correct $)$, conduction aphasia $(50.5 \%$ correct), Broca's aphasia $(41.5 \%$ correct), and Wernicke's aphasia $(26.7 \%$ correct). These differences across subtypes were significantly different based on a one-way analysis of variance (ANOVA) and LSD post-hoc comparisons (all $p<.05$ ), except for individuals with anomic and unclassifiable aphasia whose performance was statistically indistinguishable $(p=.46)$. Performance by aphasia type for the individual subtests on the repetition/span battery is shown in Table 1.

As a validation of our experimental battery, we correlated overall performance on this measure with participants' repetition subtest scores on the WAB. The relationship between the two measures was very strong, $r(84)=.95, p<.001$.

TABLE 1

Percent correct $(S D)$ on repetition/span battery by aphasia type

\begin{tabular}{lccccc}
\hline Aphasia type & Single word rep. & Pseudo word rep. & Number word rep. & Word span & Digit span \\
\hline WNL & $100(0)$ & $94(15)$ & $100(0)$ & $71(15)$ & $87(12)$ \\
Anomic & $95(13)$ & $79(24)$ & $98(8)$ & $54(14)$ & $74(16)$ \\
Unclassifiable & $93(19)$ & $67(17)$ & $95(13)$ & $51(14)$ & $69(22)$ \\
Conduction & $64(30)$ & $43(29)$ & $74(36)$ & $35(14)$ & $37(16)$ \\
Broca's & $60(32)$ & $27(29)$ & $65(31)$ & $25(13)$ & $30(15)$ \\
Wernicke's & $36(29)$ & $28(27)$ & $39(33)$ & $12(14)$ & $19(15)$ \\
Total & $85(27)$ & $69(34)$ & $87(26)$ & $51(24)$ & $65(29)$ \\
\hline
\end{tabular}

$\mathrm{WNL}=$ within normal limits on the Western Aphasia Battery; Rep. = repetition. 


\section{Anatomical correlates of AVSTM}

Overall performance (percent correct) on the repetition/span battery was associated with a network of regions in left MTG, STG (including Heschl's gyrus), and IP cortex (angular and supramarginal gyri; see Figure 2). The maximum $t$-value of 9.92 was centred in the left STG, close to the border with IP cortex (MNI coordinates -62,$42,22)$. The critical $t$-value/threshold determined by permutation testing was 4.90. All coloured voxels shown in Figure 2 as well as the following VLSM maps exceeded the threshold for significance.

Next we generated VLSM maps based on the individual subtests of the repetition/span battery. For single word repetition, the dependent variable was the maximum number of syllables in a word an examinee could repeat correctly (e.g., repeating the word corporation correctly earned a score of 4). As shown in the VLSM map in Figure 3, repetition of single words was associated primarily with left superior temporal cortex, including Heschl's gyrus. The maximum $t$-value of 7.90 was centred in the left STG, close to the border with IP cortex, in the same voxel as overall repetition/span performance above $(-62,-42,22)$. The critical $t$-value/threshold was 5.34. There was also a small number of significant voxels in IP cortex (supramarginal gyrus).

For the VLSM map of pseudoword repetition, the dependent variable was the maximum number of syllables in a pronounceable nonword that an examinee could repeat correctly (e.g., repeating molabican earned a score of 4). Accurate repetition of pseudowords was associated with a more extensive network of significant voxels that included left posterior MTG and STG (including Heschl's gyrus) and IP cortex (angular and supramarginal gyri; see Figure 4). The maximum $t$-value of 9.85 was situated at the border of STG and IP $(-62,-40,24)$, close to the location of the maximum $t$ for word repetition. The minimum $t$-value/threshold was 4.71 .

A VLSM map was generated where the dependent variable was the maximum number of syllables in a number-word that an examinee could repeat correctly (e.g., repeating six hundred eighty-nine earned a score of 6). Unlike repetition of words and pseudowords, repetition of number-words showed dependence on voxels primarily in the left mid-posterior MTG, with some extension into STG and only a few significant voxels in IP cortex (see Figure 5). The maximum $t$-value of 8.70 was centred in the

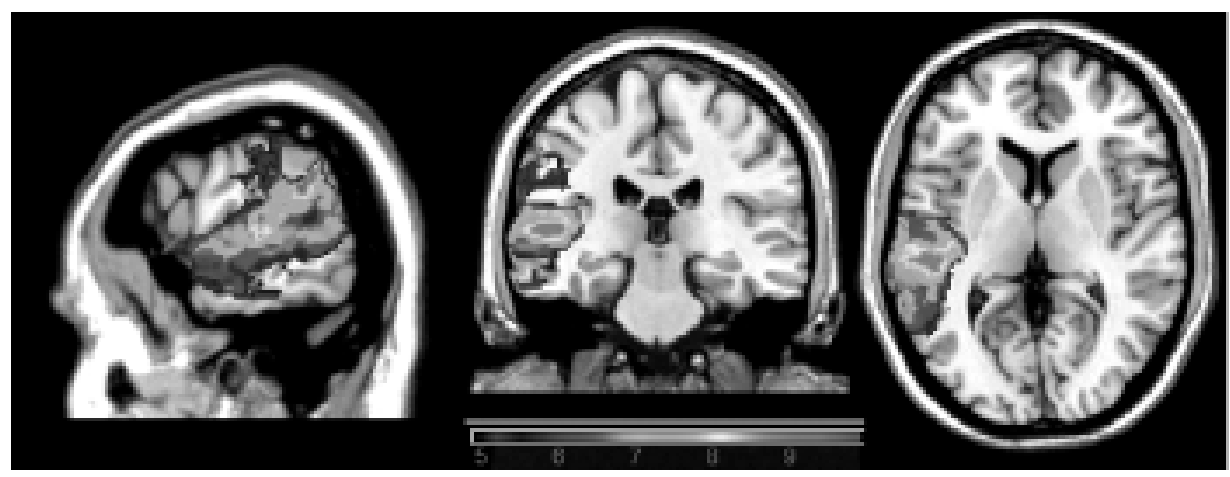

Figure 2. VLSM map of overall performance on the experimental repetition/span battery. All voxels shown in colour exceeded the critical threshold for significance, and the colours reflect increasing $t$-values from purple to red. 


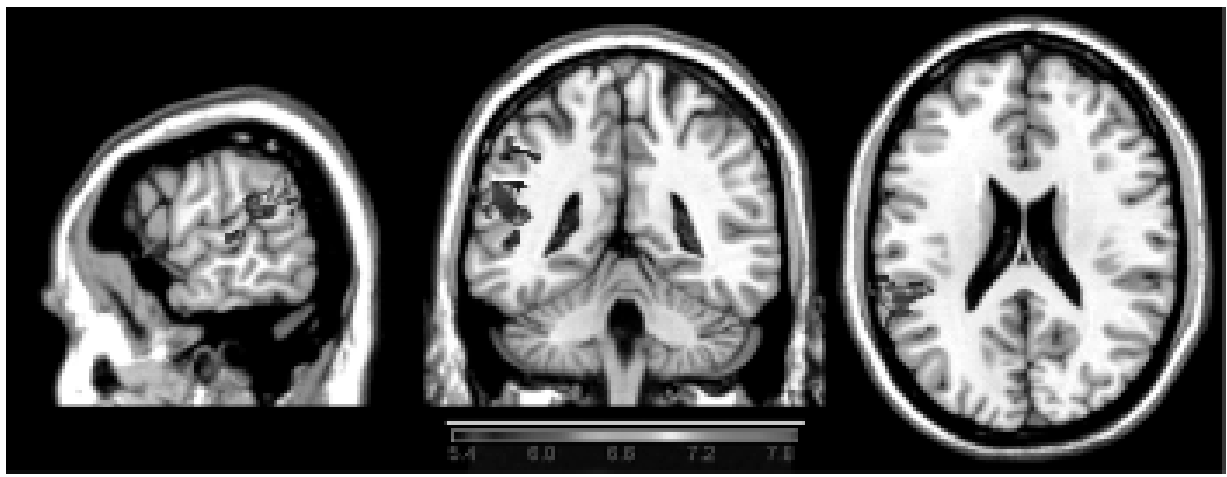

Figure 3. VLSM map showing correlates of single word repetition. Only significant voxels are shown, and the colours reflect increasing $t$-values from purple to red.

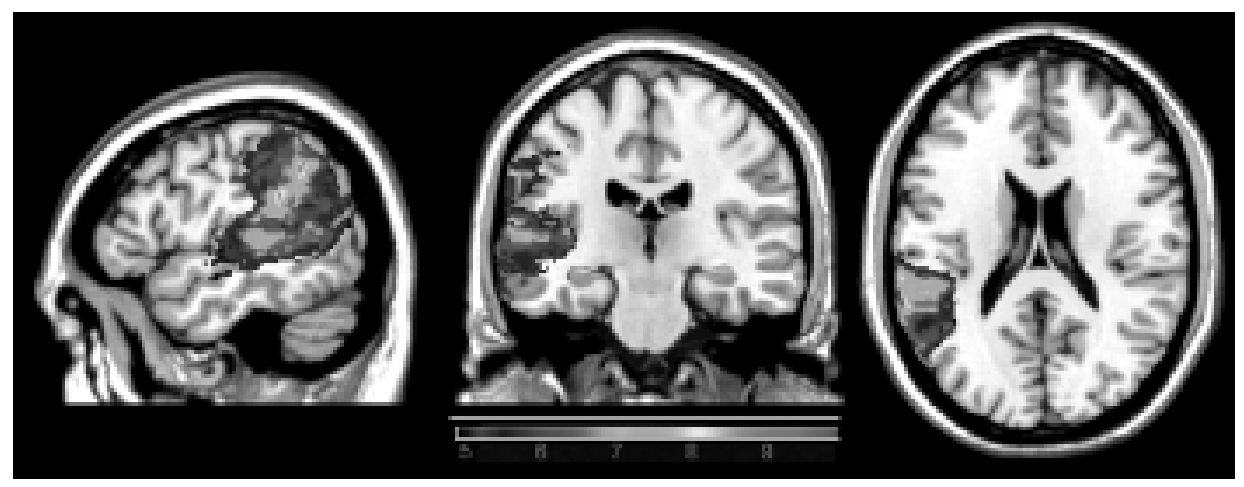

Figure 4. VLSM map showing correlates of pseudoword repetition. Only significant voxels are shown, and the colours reflect increasing $t$-values from purple to red.

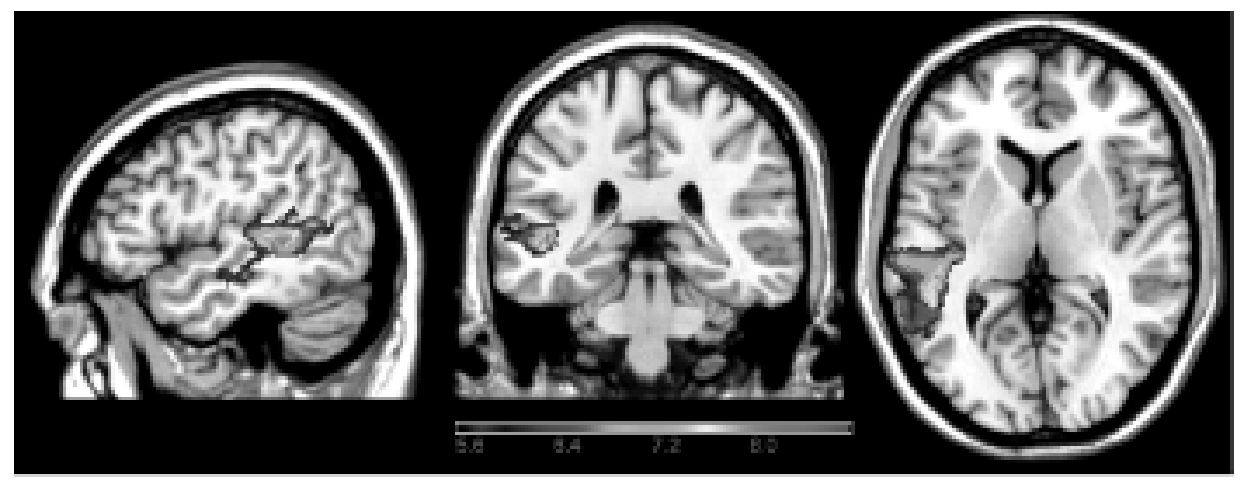

Figure 5. VLSM map showing correlates of number-word repetition. Only significant voxels are shown, and the colours reflect increasing $t$-values from purple to red. 
very medial portion of left posterior MTG, adjacent to the underlying white matter and the border with the STG $(-46,-34,4)$. The critical $t$-value was 5.61 .

The next two VLSM maps represent anatomic correlates of word and digit span, tasks that are used to measure the phonological store component of AVSTM. Word span was measured as the longest list of words that the examinee could repeat correctly in the right order (e.g., repeating car..bed..job..rule would be a word span of 4). Similarly, for digit span, the dependent variable was the longest string of digits repeated correctly (e.g., repeating 1..9..2..8..6 would be a digit span of 5). Word span performance was associated with a large portion of STG, from the superior temporal pole to posterior STG as well as Heschl's gyrus (see Figure 6). There was also extension into portions of left MTG and IP cortex (angular and supramarginal gyri). The maximum t-value of 8.25 was in the medial portion of posterior STG $(-42,-20,4)$, adjacent to the underlying white matter and Heschl's gyrus. The critical cut-off $t$-value was 4.43 .

Regions critical for digit span were very similar to those associated with word span, as well as number-word repetition (see Figure 7). The maximum t-value of 9.39 was in the medial portion of the STG, close to the border with MTG $(-46,-26,6)$, and significant regions again included a large portion of anterior-mid-posterior STG, as well as

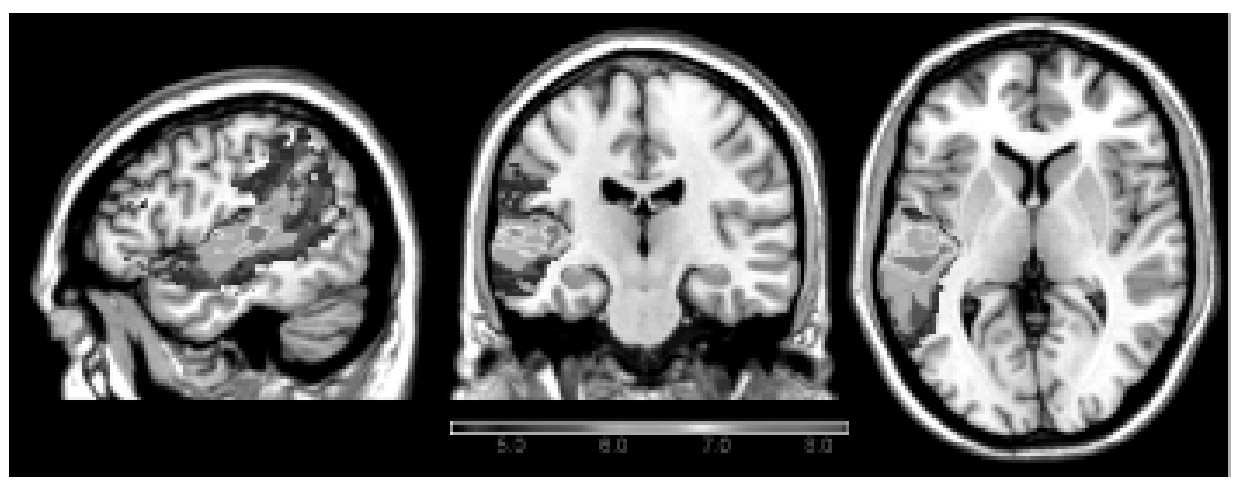

Figure 6. VLSM map showing correlates of word span performance. Only significant voxels are shown, and the colours reflect increasing $t$-values from purple to red.

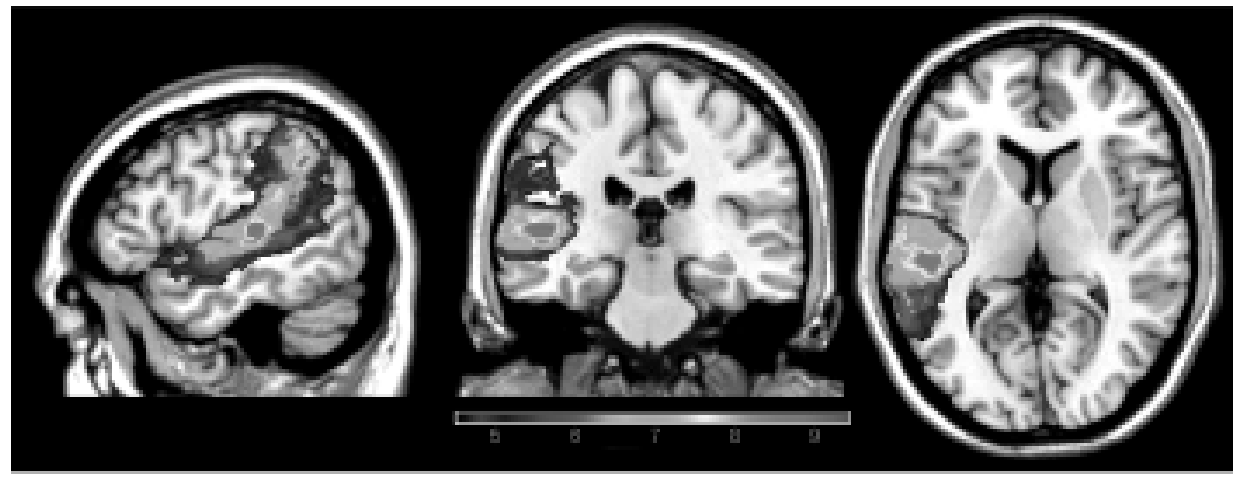

Figure 7. VLSM map showing correlates of digit span performance. Only significant voxels are shown, and the colours reflect increasing $t$-values from purple to red. 

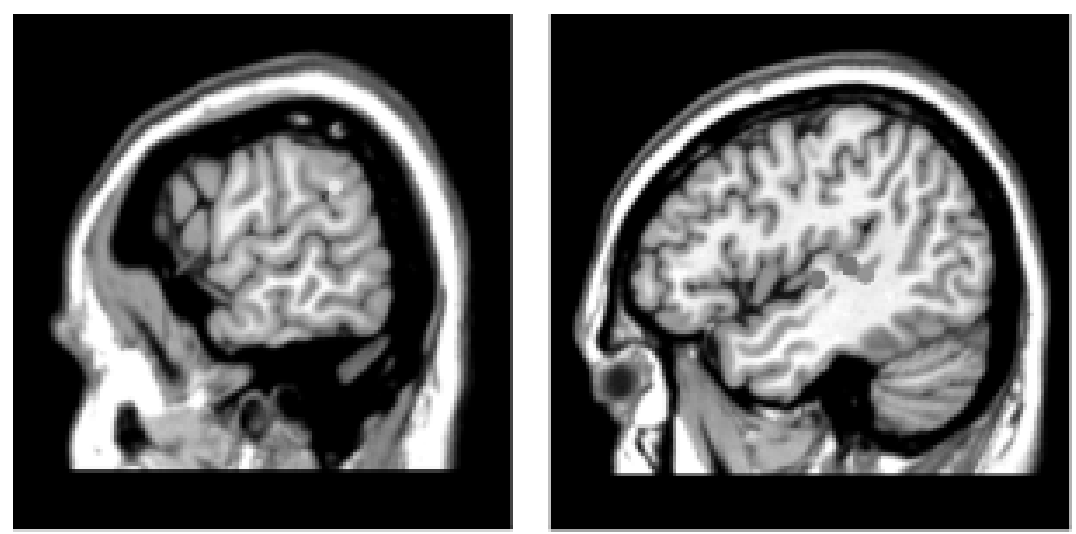

Figure 8. On the left, the maximum $t$-values are shown for single word repetition (orange) and pseudoword repetition (yellow), located at the border of left posterior STG/IP cortex. On the right, the maximum $t$ values are shown for word span (blue), digit span (red), and number-word repetition (green), located at the medial extent of the temporal gyri adjacent to underlying white matter. Note that word span is shown on the same slice as digit span and number-word repetition $(\mathrm{x}=-46)$ but was actually a few mm medial $(\mathrm{x}=-42)$.

portions of IP cortex (angular and supramarginal gyri) and mid-posterior MTG. The critical $t$ cut-off value was 4.52 .

Figure 8 shows a composite VLSM map with the locations of maximal $t$-values for all conditions. As can be seen, maximal $t$-values for word and pseudoword repetition were located adjacently in left posterior STG, on the border with IP cortex. Maximal $t$-values for word and digit span, as well as number-word repetition, were located on the border between MTG and STG and the underlying white matter.

Although the VLSM maps showed primarily cortical foci with little encroachment into white matter pathways, we further addressed the purported role of the arcuate fasciculus in repetition/span by overlaying our VLSM findings with a probabilistic diffusion tensor imaging map of the arcuate fasciculus (see Oishi et al., 2008; www.loni. ucla.edu/ICBM). As can be seen in Figure 9, the arcuate fasciculus (shown in dark yellow) was adjacent to but had minimal overlap with the regions of significance observed

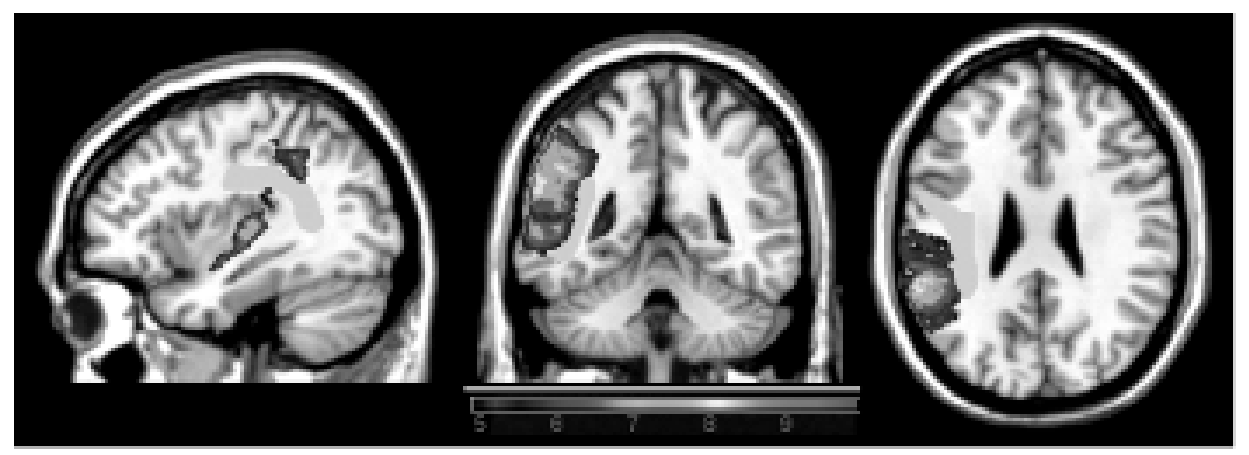

Figure 9. Probabilistic diffusion tensor imaging-based map of the arcuate fasciculus (shown in dark yellow), thresholded at $75 \%$ of cases, overlaid on the VLSM map for overall repetition/span performance. The coloured regions and legend indicate the $t$-values from the original VLSM analysis of overall repetition/span performance shown in Figure 2. 
in the VLSM map for overall repetition/span performance. It should be noted that the lack of significant VLSM findings in the arcuate fasciculus cannot be attributed to an artefact of lesion location in our participant sample, as the power analysis described above indicated adequate power for detecting significant differences in these voxels.

\section{DISCUSSION}

In the present study we examined the anatomic correlates of repetition and auditoryverbal short-term memory (AVSTM) by applying a voxel-based lesion symptom mapping (VLSM) analysis to lesion and behavioural data from a large group of individuals with left hemisphere ( $\mathrm{LH}$ ) lesions suffering from a range of aphasia severity. Individuals were tested on a series of measures that included repeating words, pseudowords, digits, and span tasks. We found that overall performance on the repetition/span battery was associated with a network of regions in the left STG and IP cortex. Counter to the classical model of repetition deficits arising from a disconnection of anterior and posterior language areas, repetition deficits across a range of stimulus types were associated with left posterior temporo-parietal cortical regions, not the arcuate fasciculus. Our other main question was whether repetition and AVSTM measures that tap the phonological store, namely digit and word span, would be associated with overlapping brain regions. There was no clear dissociation between regions associated with these two types of tasks; both repetition and the phonological store tasks relied heavily on left mid-posterior STG, as well as portions of MTG and IP cortex.

Analysis of individual repetition/span subtests showed some distinctions in the neural correlates across conditions. While word and pseudoword repetition had similar maximal values in posterior STG, pseudoword repetition was associated with a much larger region of STG, with significant extension into IP cortex. It is likely that pseudoword repetition is dependent on a larger region of STG/IP cortex because the task is not overlearned like simple word repetition and cannot be supported by other intact brain regions involved in semantics. In contrast to single word and pseudoword repetition, word and digit span were associated with maximal values that were located on the boundary of left STG, adjacent to the underlying white matter and adjacent to Heschl's gyrus. The VLSM maps of word and digit span were very similar, with significant regions including portions of left mid to posterior MTG and STG, as well as IP cortex. Interestingly, though, both of the measures involving numbers (digit span and number-word repetition) had maximal $t$-values in adjacent cortex on the medial portion of the MTG/STG border. Last, unlike the other repetition and STM measures, the repetition of number-words was almost entirely dependent on regions within the left MTG.

Our findings are broadly consistent with other lesion studies suggesting that the phonological store component of AVSTM that subserves performance on tasks such as digit and word span is dependent on left STG/IP (e.g., Baldo \& Dronkers, 2006; Baldo, Klosterman, \& Dronkers, 2008; Hickok \& Poeppel, 2004; Leff et al., 2009; Warrington et al., 1971). Similar to the current study, Leff et al. (2009) used a voxelbased analysis in a large sample and found that digit span performance was most dependent on the posterior superior temporal gyrus. Baldo et al. (2008) showed that individuals with STG/IP lesions were impaired on a sentence repetition recognition task, in which participants had to point to one of three sentences that matched a sentence they just heard. Interestingly, the individuals with STG/IP lesions performed 
like control participants when targets and distractors were semantically distinct, but when the targets and distractors were semantically congruent they could not distinguish the target sentence. That is, individuals with STG/IP lesions could process and hold on to the semantic content of sentences but could not hold on to the verbatim trace (see also Butterworth, Campbell, \& Howard, 1986; Martin, Shelton, \& Yaffee, 1994; Saffran \& Marin, 1975). Like the early STM cases of Warrington and colleagues, these findings showed that repetition deficits following STG/IP damage are not simply due to disturbed output or paraphasic errors, as the deficit is apparent when participants respond with a pointing response; rather the verbatim trace in the phonological store is not maintained/accessible. Such findings are consistent with the fact that these individuals have relatively good comprehension abilities, as verbatim trace information is not generally critical for understanding conversational speech. However, when verbatim information is required (e.g., when rehearsing a phone number or understanding complex sentences), these individuals' performance breaks down (Dronkers et al., 2004).

The current findings are also consistent with functional imaging studies in healthy participants, which have generally implicated left STG and inferior IP in the brief storage of verbal information (Awh, Smith, \& Jonides, 1995; Henson, Burgess, \& Frith, 2000; Honey et al., 2000; Jonides et al., 1998; Paulesu, Frith, \& Frackowiak, 1993 Salmon et al., 1996; but see Chein \& Fiez, 2001; Gruber, 2001). Recently, Acheson, Hamidi, Binder, and Postle (2010) carried out a combined fMRI/rTMS study comparing regions critical for AVSTM (using nonwords) versus lexical-semantic retrieval (picture naming). Like the current study, they found that the posterior STG was critical for AVSTM, while picture naming relied on the MTG. Buchsbaum et al. (2011) did a meta-analysis of functional imaging studies of phonological STM and overlapped those regions of activation with lesions seen in a group of individuals with conduction aphasia. The brain region critical to both was the left posterior planum temporale, which is on the posterior, superior plane of the STG. In an earlier paper Buchsbaum, Olsen, Koch, and Berman (2005) argued that this region is critical to maintenance of information regardless of stimulus modality, whereas the left STG/STS was critical when items were delivered auditorily.

Both prior lesion and functional imaging studies have suggested that the articulatory rehearsal component of AVSTM, tapped by tasks with delayed response/ manipulation of verbal information, is more dependent on left inferior lateral prefrontal cortex (Baldo \& Dronkers, 2006; D’Esposito \& Postle, 1999). In the current study we did not expect to see significant voxels in these regions because our tasks did not require such manipulation but rather relied primarily on the phonological store. Previous work from our lab has shown that individuals with focal IF lesions do indeed have difficulty with STM tasks that involve manipulation and/or long delay periods (Baldo \& Dronkers, 2006; Baldo \& Shimamura, 2000).

The current study was limited in that we were not able to test the role of the right hemisphere (RH) in AVSTM, due to the fact that only a minority of individuals in our participant pool had RH lesions. A number of studies have found that the RH is most critical for spatial working memory (e.g., Smith et al., 1995) but that it may also play a role in AVSTM under certain conditions (Ravizza, Behrmann, \& Fiez, 2005; Salmon et al., 1996). The current battery also did not allow us to assess brain regions involved in non-verbal STM, for example, using spatial span/working memory tasks. We have completed such testing in a smaller sub-sample of individuals (Baldo \& Dronkers, 2006) and found that individuals with focal left IP lesions had reduced 
spatial spans relative to controls (e.g., on the Corsi blocks task); however, this may have been due to a covert verbal strategy used by controls. Lastly, the VLSM analyses in the current study were confined to those voxels in which there was adequate lesion coverage. Thus the role of regions such as fronto-polar, ventral temporal, and occipital cortex could not be explored with the current dataset. Nonetheless we had adequate statistical power in those regions that were the basis of our predictions, namely left temporo-parietal cortex and the arcuate fasciculus.

The current findings have implications for our understanding of repetition deficits in aphasia. It is likely that the reason repetition deficits are commonly associated with conduction aphasia, even though disturbed repetition occurs in other forms of aphasia such as Wernicke's aphasia, is that reduced repetition is often the most striking deficit in these individuals. For example, one of our current participants with conduction aphasia is able to carry on a casual conversation quite fluently but when asked to repeat a list of numbers or words verbatim he is suddenly halting in his output and appears non-fluent. Such individuals tell us that they hear what we say, but then they feel as if the information is "just gone" from their minds. In contrast, individuals with Wernicke's aphasia have such disturbed speech output and comprehension difficulties that the repetition deficits are not the most striking deficit and do not seem of paramount importance. Anatomically this is consistent with our findings, because individuals with conduction aphasia have relatively circumscribed lesions involving the regions implicated in the current study, namely left STG and IP (Dronkers \& Baldo, 2009). In contrast, individuals with Wernicke's aphasia have large left temporal lobe lesions that encompass not only these temporo-parietal regions but also left MTG, which likely accounts for their impaired lexical-semantic processing/comprehension as well (Acheson et al., 2010; Baldo, Arevalo, Wilkins, \& Dronkers, 2009; Dronkers \& Baldo, 2009; Dronkers et al., 2004).

In conclusion, VLSM analysis of a large sample of individuals with aphasia found that the anatomical regions most critical to repetition and the phonological store component of AVSTM are located within left temporo-parietal cortex, not white matter pathways such as the arcuate fasciculus. More specifically, the VLSM analysis showed that single word repetition, including pseudoword repetition, is most dependent on the left posterior STG, on the border with IP cortex, and that the phonological store, as measured by word and digit span, relies most heavily on the medial portion of the MTG/STG border, adjacent to the underlying white matter and Heschl's gyrus. These findings reinforce the notion that repetition/AVSTM deficits can be seen across a wide range of individuals with aphasia, depending on the site of injury.

\section{REFERENCES}

Acheson, D. J., Hamidi, M., Binder, J. R., \& Postle, B. R. (2010). A common neural substrate for language production and verbal working memory. Journal of Cognitive Neuroscience, ??, ???-???.

Anderson, J. M., Gilmore, R., Roper, S., Crosson, B., et al. (1999). Conduction aphasia and the arcuate fasciculus: A reexamination of the Wernicke-Geschwind model. Brain and Language, 70, 1-12.

Awh, E., Smith, E., \& Jonides, J. (1995). Human rehearsal processes and the frontal lobes: PET evidence. Annals of the New York Academy of Sciences, 769, 97-117.

Axer, H., Keyserlingk, A., Berks, G., \& Keyserlingk, D. (2001). Supra- and infra-sylvian conduction aphasia. Brain and Language, 76, 317-331.

Baddeley, A. (2000). The episodic buffer: A new component of working memory? Trends in Cognitive Sciences, 4, 417-423.

Baddeley, A. (2003). Working memory: Looking back and looking forward. Nature Reviews Neuroscience, $4,829-839$. 
Baddeley, A., \& Hitch, G. (1994). Developments in the concept of working memory. Neuropsychology, 8, 485-493.

Baldo, J., \& Dronkers, N. (2006). The role of inferior frontal and inferior parietal cortex in working memory. Neuropsychology, 20, 529-538.

Baldo, J., Klosterman, E., \& Dronkers, N. (2008). It's either a cook or a baker: Patients with conduction aphasia get the gist but lose the trace. Brain and Language, 105, 134-140.

Baldo, J., Wilson, S., \& Dronkers, N. (in press). Uncovering the neural substrates of language: A voxel-based lesion symptom mapping approach. In M. Faust (Ed.), Advances in the neural substrates of language: Toward a synthesis of basic science and clinical research. ?????????????????

Baldo, J. V., Arevalo, A., Wilkins, D. P., \& Dronkers, N. (2009). Voxel-based lesion analysis of categoryspecific naming on the Boston Naming Test. Center for Research in Language Technical Report, University of California, San Diego, 21, 1-12.

Baldo, J. V., \& Shimamura, A. P. (2000). Spatial and color working memory in patients with lateral prefrontal cortex lesions. Psychobiology, 28, 156-167.

Bartha, L., \& Benke, T. (2003). Acute conduction aphasia: An analysis of 20 cases. Brain and Language, 85, 93-108.

Bates, E., Wilson, S., Saygin A. P., Dick, F., Sereno, M., Knight, R. T., et al. (2003). Voxel-based lesionsymptom mapping. Nature Neuroscience, 6, 448-450.

Belleville, S., Peretz, I., \& Arguin, M. (1992). Contribution of articulatory rehearsal to short-term memory: Evidence from a case of selective disruption. Brain and Language, 43, 713-746.

Benson, D. F., Sheremata, W. A., Bouchard, R., Segarra, J. M., Price, D., \& Geschwind, N. (1973). Conduction aphasia: A clinicopathological study. Archives of Neurology, 28, 339-346.

Bernal, B., \& Ardila, A. (2009). The role of the arcuate fasciculus in conduction aphasia. Brain, 132, 2309-2316.

Breier, J. I., Hasan, K. M., Zhang, W., Men, D., \& Papanicolaou, A. C. (2008). Language dysfunction after stroke and damage to white matter tracts evaluated using diffusion tensor imaging. American Journal of Neuroradiology, 29, 483-487.

Brett, M., Leff, A. P., Rorden, C., \& Ashburner, J. (2001). Spatial normalization of brain images with focal lesions using cost function masking. Neuroimage, 14, 486-500.

Brown, J. W. (1975). The problem of repetition: A study of "conduction" aphasia and the "isolation" syndrome. Cortex, 11, 37-52.

Buchsbaum, B. R., Baldo, J. V., Okada, K., Berman, K. F., Dronkers, N. F., D’Esposito, M., et al. (2011). Conduction aphasia, sensory-motor integration, and phonological short-term memory - an aggregate analysis of lesion and fMRI data. Brain and Language, ??, ???-???

Buchsbaum, B. R., Olsen, R. K., Koch, P., \& Berman, K. F. (2005). Human dorsal and ventral auditory streams subserve rehearsal-based and echoic processes during verbal working memory. Neuron, 48, 687-697.

Butterworth, B., Campbell, R., \& Howard, D. (1986). The uses of short-term memory: A case study. Quarterly Journal of Experimental Psychology, 38A, 705-737.

Caramazza, A., Basili, A. G., Koller, J. J., \& Berndt, R. S. (1981). An investigation of repetition and language processing in a case of conduction aphasia. Brain and Language, 14, 235-271.

Catani, M., Jones, D. K., \& Ffytche, D. H. (2005). Perisylvian language networks of the human brain. Annals of Neurology, 57, 8-16.

Catani, M., \& Mesulam, M. (2008). The arcuate fasciculus and the disconnection theme in language and aphasia: History and current state. Cortex, 44, 953-961.

Chein, J. M., \& Fiez, J. A. (2001). Dissociation of verbal working memory system components using a delayed serial recall task. Cerebral Cortex, 11, 1003-1014.

Cohen, J. (1988). Statistical power analysis for the behavioural sciences (2nd ed.). Hillsdale, NJ: Lawrence Erlbaum Associates Inc.

Cohen, J. (1992). A power primer. Psychological Bulletin, 112, 155-159.

Cohen, J. D., Perlstein, W. M., Braver, T. S., Nystrom, L. E., Noll, D. C., Jonides, J., et al. (1997). Temporal dynamics of brain activation during a working memory task. Nature, 386, 604-608.

Damasio, H., \& Damasio, A. (1980). The anatomical basis of conduction aphasia. Brain, 103, 337-350.

D'Esposito, M., Detre, J. A., Alsop, D. C., Shin, R. K., Atlas, S., \& Grossman, M. (1995). The neural basis of the central executive system of working memory. Nature, 378, 279-281.

D'Esposito, M., \& Postle, B. R. (1999). The dependence of span and delayed-response performance on prefrontal cortex. Neuropsychologia, 37, 1303-1315. 
Dronkers, N. F., \& Baldo, J. V. (2009). Language: Aphasia. In L. R. Squire (Ed.), The new encyclopedia of neuroscience (pp. 343-348). Oxford, UK: Elsevier.

Dronkers, N. F., Wilkins, D. P., Van Valin, R. D. Jr., Redfern, B. B., \& Jaeger, J. J. (2004). Exploring brain areas involved in language comprehension using a new method of lesion analysis. Cognition, 92, 145-177.

Duffau, H., Capelle, L., Sichez, N., Denvil, D., Lopes, M., Sichez, J-P., et al. (2002). Intraoperative mapping of the subcortical language pathways using direct stimulations: An anatomo-functional study. Brain, $125,199-214$.

Fridriksson, J., Kjartansson, O., Morgan, P. S., Hjaltason, H., Magnusdottir, S., Bonilha, L., et al. (2010). Impaired speech repetition and left parietal lobe damage. Journal of Neuroscience, 30, 11057-11061.

Geschwind, N. (1972). Language and the brain. Scientific American, 226, 76-83.

Goodglass, H. (1992). Diagnosis of conduction aphasia. In Conduction aphasia. Hillsdale, NJ: Lawrence Erlbaum Associates Inc.

Green, E., \& Howes, D. H. (1978). The nature of conduction aphasia: A study of anatomic and clinical features and of underlying mechanisms. In H. Whitaker \& H. A. Whitaker (Eds.), Studies in neurolinguistics. New York: Academic Press.

Gruber, O. (2001). Effects of domain-specific interference on brain activation associated with verbal working memory task performance. Cerebral Cortex, 11, 1047-1055.

Henson, R. N., Burgess, N., \& Frith, C. D. (2000). Recoding, storage, rehearsal and grouping in verbal short-term memory: An fMRI study. Neuropsychologia, 38, 426-440.

Hickok, G., Buchsbaum, B., Humphries, C., \& Muftuler, T. (2003). Auditory-motor integration revealed by fMRI: Speech, music, and working memory in area Spt. Journal of Cognitive Neuroscience, 15, 673-682.

Hickok, G., \& Poeppel, D. (2004). Dorsal and ventral streams: A framework for understanding aspects of the functional anatomy of language. Cognition, 92, 67-99.

Honey, G. D., Bullmore, E. T., \& Sharma, T. (2000). Prolonged reaction time to a verbal working memory task predicts increased power of posterior parietal cortical activation. NeuroImage, 12, 495-503.

Jonides, J., Schumacher, E. H., Smith, E. E., Koeppe, R. A., Awh, E., Reuter-Lorenz, P. A., et al. (1998). The role of parietal cortex in verbal working memory. Journal of Neuroscience, 18, 5026-5034.

Kempler, D., Metter, E. J., et al. (1988). Disconnection and cerebral metabolism: The case of conduction aphasia. Archives of Neurology, 45, 275-279.

Kertesz, A. (1982). Western Aphasia Battery. New York: Grune \& Stratton.

Kimberg, D. Y., Coslett, H. B., \& Schwartz, M. F. (2007). Power in voxel-based lesion-symptom mapping. Journal of Cognitive Neuroscience, 19, 1067-1080.

Kohler, K., Bartels, C., Herrmann, M., Dittmann, J., \& Wallesch, C-W. (1998). Conduction aphasia 11 classic cases. Aphasiology, 12, 865-884.

Leff, A. P., Schofield, T. M., Crinion, J. T., Seghier, M. L., Grogan, A., Green, D. W., et al. (2009). The left superior temporal gyrus is a shared substrate for auditory short-term memory and speech comprehension: evidence from 210 patients with stroke. Brain, ??, ???-???.

Martin, N., \& Ayala, J. (2004). Measurements of auditory-verbal STM span in aphasia: Effects of item, task, and lexical impairment. Brain and Language, 89, 464-483.

Martin, R. C., Shelton, J. R., \& Yaffee, L. S. (1994). Language processing and working memory: Neuropsychological evidence for separate phonological and semantic capacities. Journal of Memory and Language, 33, 83-111.

Mendez, M. F., \& Benson, F. (1985). Atypical conduction aphasia: A disconnection syndrome. Archives of Neurology, 42, 886-891.

Oishi, K., Zilles, K., Amunts, K., et al. (2008). Human brain white matter atlas: Identification and assignment of common anatomical structures in superficial white matter. NeuroImage, 43, 447-457.

Owen, A. M., McMillan, K. M., Laird, A. R., \& Bullmore, E. (2005). N-back working memory paradigm: A meta-analysis of normative functional neuroimaging studies. Human Brain Mapping, 25, 46-59.

Paulesu, E., Frith, C., \& Frackowiak, D. (1993). The neural correlates of the verbal component of working memory. Nature, 362, 342-345.

Petrides, M., Alivisatos, B., Meyer, E., \& Evans, A. C. (1993). Functional activation of the human frontal cortex during the performance of verbal working memory tasks. Proceedings of the National Academy of Sciences USA, 90, 878-882.

Petrides, M., \& Pandya, D. N. (1988). Association fiber pathways to the frontal cortex from the superior temporal region in the rhesus monkey. Journal of Comparative Neurology, 273, 52-66. 
Postle, B. R., Berger, J. S., \& D’Esposito, M. D. (1999). Functional neuroanatomical double dissociation of mnemonic and executive control processes contributing to working memory performance. Proceedings of the National Academy of Sciences USA, 96, 12959-12964.

Quigg, M., \& Fountain, N. B. (1999). Conduction aphasia elicited by stimulation of the left posterior superior temporal gyrus. Journal of Neurology, Neurosurgery, \& Psychiatry, 66, 393-396.

Quigg, M., Geldmacher, D. S., \& Elias, W. J. (2006). Conduction aphasia as a function of the dominant posterior perisylvian cortex. Journal of Neurosurgery, 104, 845-848.

Raven, J. (1962). Coloured Progressive Matrices. New York: The Psychological Corporation.

Ravizza, S. M., Behrmann, M., \& Fiez, J. A. (2005). Right parietal contributions to verbal working memory: Spatial or executive. Neuropsychologia, 43, 2057-2067.

Ravizza, S. M., Delgado, M. R., Chein, J. M., Becker, J. T., \& Fiez, J. A. (2004). Functional dissociations within the inferior parietal cortex in verbal working memory. Neuroimage, 22, 562-573.

Rorden, C., \& Brett, M. (2000). Stereotaxic display of brain lesions. Behavioural Neurology, 12, 191-200.

Saffran, E. M., \& Marin, O. S. M. (1975). Immediate memory for word lists and sentences in a patient with deficient auditory short-term memory. Brain and Language, 2, 420-433.

Salmon, E., Van der Linden, M., Collette, F., Delfiore, G., Maquet, P., Degueldre, C., et al. (1996). Regional brain activity during working memory tasks. Brain, 119, 1617-1625.

Selnes, O. A., van Zijl, P. C., Baker, P. B., Hillis, A. E., \& Mori, S. (2002). MR diffusion tensor imaging documented arcuate fasciculus lesion in a patient with normal repetition performance. Aphasiology, 16, 897-902.

Shallice, T., \& Warrington, E. K. (1977). Auditory-verbal short-term memory impairment and conduction aphasia. Brain and Language, 4, 479-491.

Shuren, J. E., Schefft, B. K., Yeh, H-S., Privitera, M. D., Cahill W. T., \& Houston, W. (1995). Repetition and the arcuate fasciculus. Journal of Neurology, 242, 596-598.

Smith, E. E., Jonides, J., Koeppe, R. A., Awh, E., Schumacher, E. H., \& Minoshima, S. (1995). Spatial versus object working memory: PET investigations. Journal of Cognitive Neuroscience, 7, 337-356.

Turken, A., \& Dronkers, N. F. (2011). The neural architecture of the language comprehension network: Converging evidence from lesion and connectivity analyses. Frontiers in Systems Neuroscience, $5,1$. Doi: $10.3389 /$ fnsys.2011.00001

Vallar, G., \& Baddeley, A. (1984). Fractionation of working memory: Neuropsychological evidence for a phonological short-term store. Journal of Verbal Learning and Verbal Behavior, 233, 151-161.

Vallar, G., Di Betta, A. M., \& Silveri, C. (1997). The phonological short-term store-rehearsal system: Patterns of impairment and neural correlates. Neuropsychologia, 35, 795-812.

Warrington, E., \& Shallice, T. (1969). The selective impairment of auditory verbal short-term memory. Brain, 92, 885-896.

Warrington, E. K., Logue, V., \& Pratt, R. T. (1971). The anatomical localization of selective impairment of auditory verbal short-term memory. Neuropsychologia, 9, 377-387.

Wernicke, K. (1906). The aphasia symptom complex [translated by G. H. Eggert (1977), Wernicke's works on aphasia: A sourcebook and review]. The Hague: Mouton Publishers. 